\title{
Behavior Fusion of Robot Navigation Using a Fuzzy Neural Network
}

\author{
Kai-Tai Song and Jean-Yuan Lin
}

\begin{abstract}
This paper presents a design of behaviorfusion architecture for mobile robot navigation. We first design three behaviors for robot navigation, including obstacle avoidance, wall following, and goal seeking. We implement these primitive behaviors by using fuzzy-logic control approaches. Then, the fusion weight of each behavior is determined by using the proposed behaviorfusion neural network. The neural network maps the current environment sensor data to suitable fusion weights. Both computer simulation and practical experiments verify the effectiveness of the method.
\end{abstract}

\section{INTRODUCTION}

$\mathrm{B}$ ehavior-based architectures have been widely used for navigation control of mobile robots[1-2], because these architectures facilitate a mobile robot to react in real time to the environmental information. With behavior-based approaches, one decomposes complex navigation tasks into several, well-specified behaviors that are easier to design and carry out. In [2], robotic behavior is defined such that a behavior is a control law that satisfies a set of constraints to achieve and maintain a particular goal. Thus when a behavior is activated, the output of the behavior never takes into account of other behaviors, each behavior is designed to achieve a specified navigation task. However, it also means that there may have conflicts among the outputs of different behaviors. For more practical applications, the environment as well as the task will become more complex. The coordination of behavior sets becomes important. Many behavior coordination and fusion methods have been studied in recent years[3-7]. Basically, behavior coordination can be categorized into two groups, namely behavior arbitration and behavior fusion. In the former, only one behavior's output will be valid at any one time according the sensory inputs [1]. While for the latter, each behavior can have the opportunity to contribute the control output, depending on the sensory information. The problem of behavior fusion then becomes to determine the participation weight of each individual behavior.

This paper studies a fuzzy-neuro design for determining the fusion weights of several navigation behaviors. In this design, a mapping between fusion weights and the immediate environmental configuration is generated for providing the proper fusion weights efficiently. The purpose is to achieve autonomous navigation in a dynamic changing environment. We first designed three primitive behaviors for autonomous navigation in an indoor environment. These behaviors are

This work was supported by the National Science Council of Taiwan R.O.C., under grant. NSC-94-22 18-E-009-008.

Kai-Tai Song and Jean-Yuan Lin are with the Department of Electrical and Control Engineering, National Chiao Tung University, Hsinchu, Taiwan R.O.C. (e-mail: ktsong@mail.nctu.edu.tw, marksman.ece92g@nctu.edu.tw). goal seeking behavior, wall following behavior, and obstacle avoidance behavior. Each behavior is designed by using simple fuzzy logic control approach to achieve respective navigation task. The output of fuzzy logic controller of each behavior reacts to environmental information from ultrasonic sensors and the motor shaft encoder signals.

\section{FUZZY BEHAVIORS}

A mobile robot equipped with ultrasonic sensors has been designed and constructed as shown in Fig.1. The mobile robot has two independent drive wheels and two casters for balance. Robot motion is controlled by adjusting the velocities of two driving wheels. The velocity servo control is handled by a self-made two-axis motion control card based on a TMS320F240 DSP chip. The ultrasonic sensors can measure distance up to five meters with $4 \mathrm{~Hz}$ for one scanning of all twelve sensors on board. In this study, we use only the front nine sensors for navigation design. The output of the fuzzy logic controller, which implements each behavior, is designed to change the moving direction of the mobile robot. We suppose that the mobile robot moves forward with a constant linear speed. If the output of fuzzy logic controller, i.e. the speed difference of two drive wheels, of a behavior is zero, the mobile robot will then move straight forward.

Fuzzy systems have the capacity to handle uncertain and imprecise information using linguistic rules. When a mobile robot navigates in an unstructured environment, it needs to detect its surrounding environment and interprets the gathered environmental information. From sensory information, the robot can obtain the knowledge of its position and the distance to surrounding obstacles. Fuzzy logic control (FLC) have been widely used for mobile robot navigation [8-12], mainly due to FLC is capable of offering inference using environmental data, even under motion and sensor uncertainties. We designed three primitive behaviors by using FLC. The inputs of each FLC are distances measured by ultrasonic sensors, and the output is the differential speed between left and right drive wheels $(\mathrm{cm} / \mathrm{s})$. We divided nine front-side sonar sensors into five groups to detect the distance from surrounding obstacles as shown in Fig.2. In each group the minimum sensor value is taken as the distance from obstacles in five directions as shown in Fig. 3. In the following, these three behaviors are described.

\section{A. Obstacle Avoidance Behavior}

We used three fuzzy rules to implement the obstacle avoidance behavior. They are listed as follows.

a. If the distance from obstacle is close, then the differential speed is big.

b. If the distance from obstacle is medium, then differential speed is medium. 
c. If the distance from obstacle is far, then the differential speed is small.

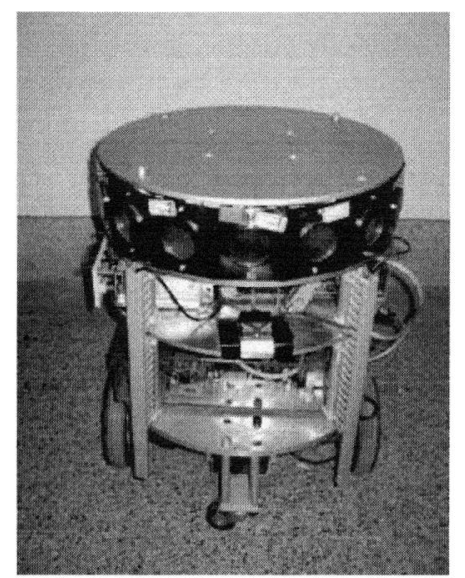

Fig.1. The experimental mobile robot equipped with a ring of twelve ultrasonic sensors.

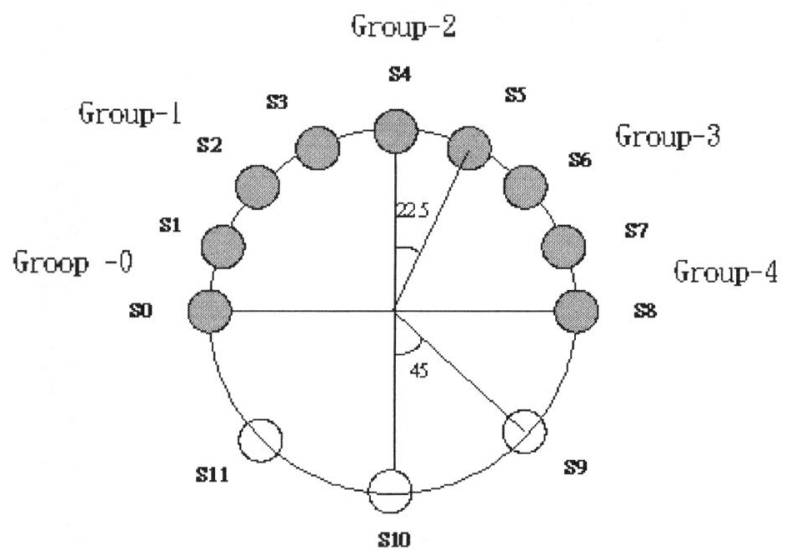

Fig. 2. Numbering of the ultrasonic sensors.

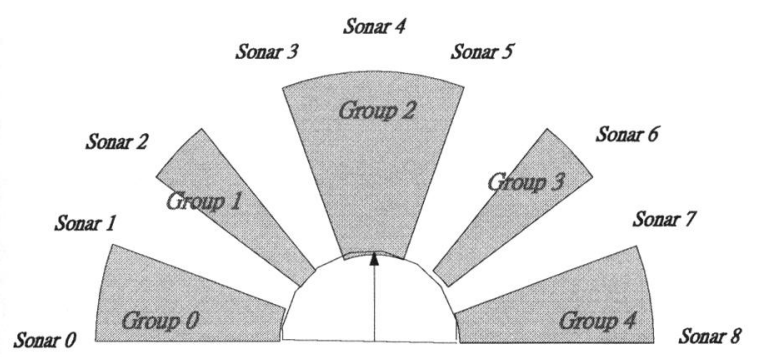

Fig. 3. Grouping of the ultrasonic sensors on the mobile robot.
Fig. 4 shows the fuzzy logic design of obstacle avoidance behavior. The front side sensors S3 S5 (Group 2) are used in this behavior as input to the FLC. Furthermore, we use the distance information from ultrasonic sensor 2 and ultrasonic sensor 6 (see Fig. 5)to decide which side that robot should choose to avoid the obstacle, which is in front of the moving direction of the robot. The membership functions of input and output fuzzy variables are illustrated in Fig. 6. One can see that by using only three fuzzy rules, the differential speed between left and right wheels can be determined for obstacle avoidance. The overall input-output relation of obstacle avoidance behavior is shown in Fig. 7.

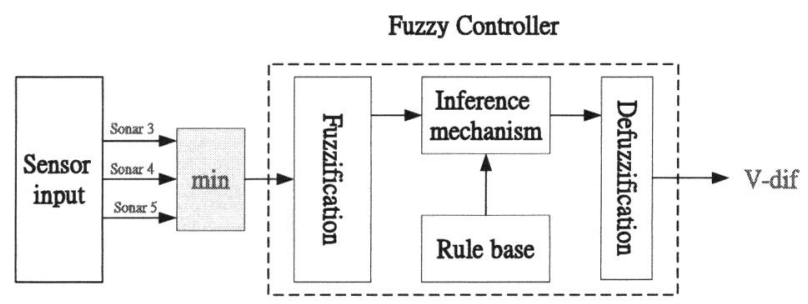

Fig. 4. Fuzzy logic design of obstacle avoidance behavior

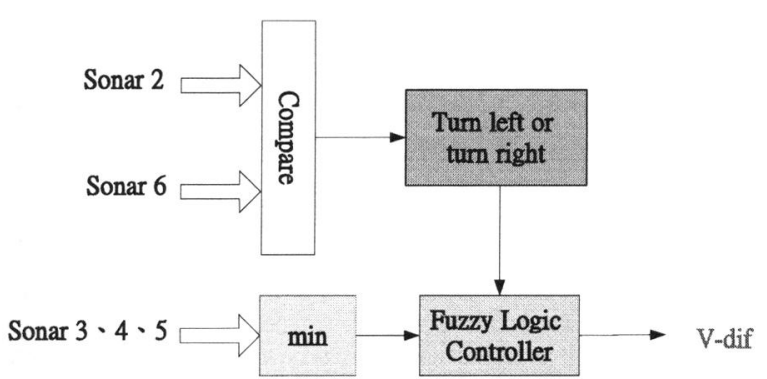

Fig. 5. Input and output of the obstacle avoidance behavior.

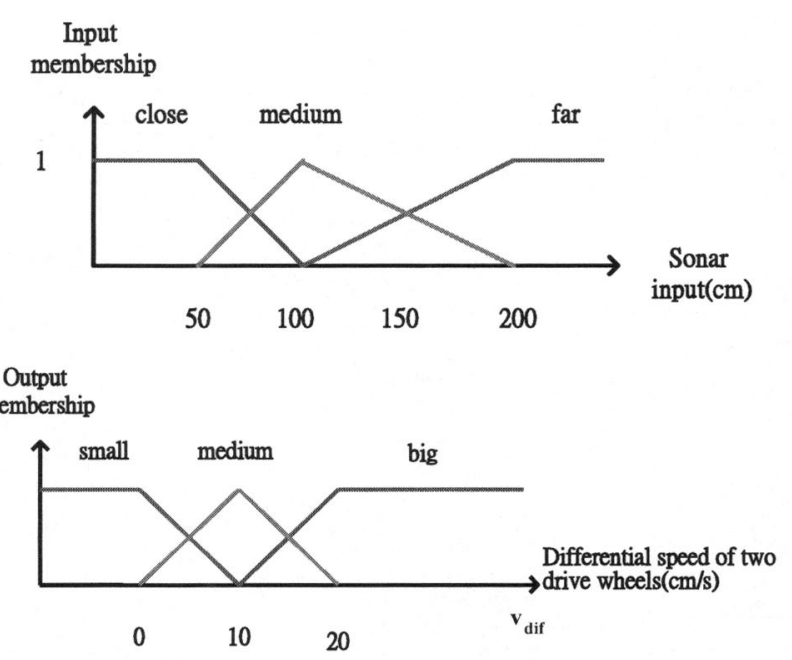

Fig. 6. The membership function of obstacle avoidance FLC. 


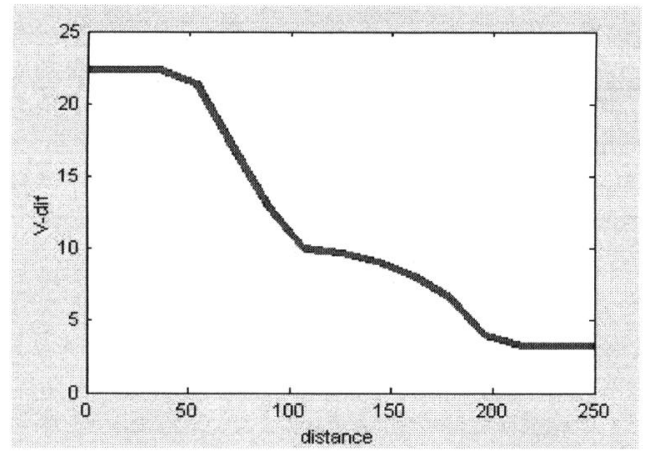

Fig. 7. Input /output relation of obstacle avoidance FLC.

\section{B. Goal Seeking Behavior}

In this design, the behavior of goal seeking is treated as trying to move along the direction to the target. Using the definition, we define the relative angle between the robot heading and goal direction as the input of the goal seeking FLC behavior. As illustrated in Fig. 8, the relative angle to the goal is used to determine the differential speed of mobile robot by using fuzzy inference. The fuzzy rules are as follows.

a. If the included angle is big, then the differential speed is big.

b. If the included angle is medium, then the differential speed is medium.

c. If the included angle is small, then the differential speed is zero.

The rules are used to modulate the heading of mobile robot. Let the robot move along the direction to the target. Its membership function of rules is displayed in the Fig. 9. The input and output relation of the goal seeking FLC is shown in Fig. 10. One can see that when the angle to the target direction increases, the differential speed between two driver wheels increases, too.

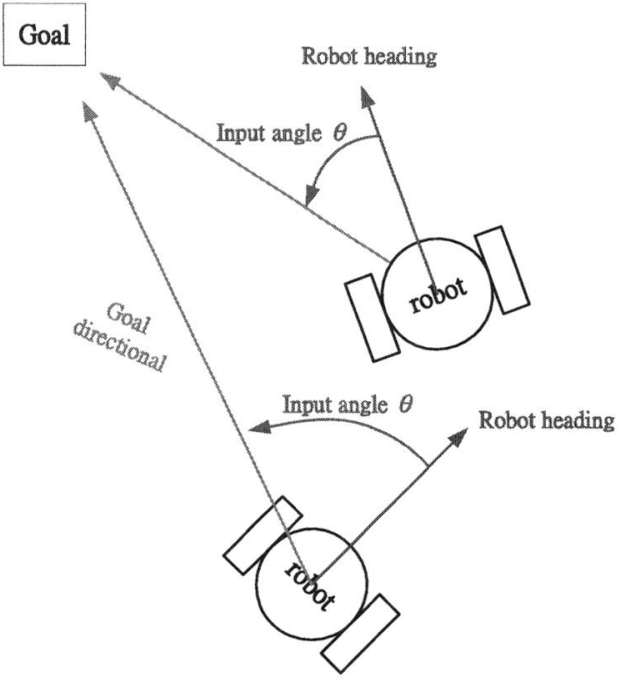

Fig. 8. The relative angle to the goal.

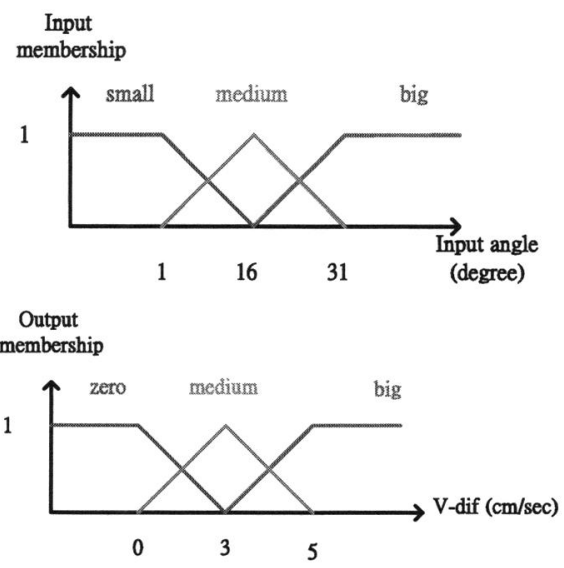

Fig. 9. Membership function of the goal seeking FLC.

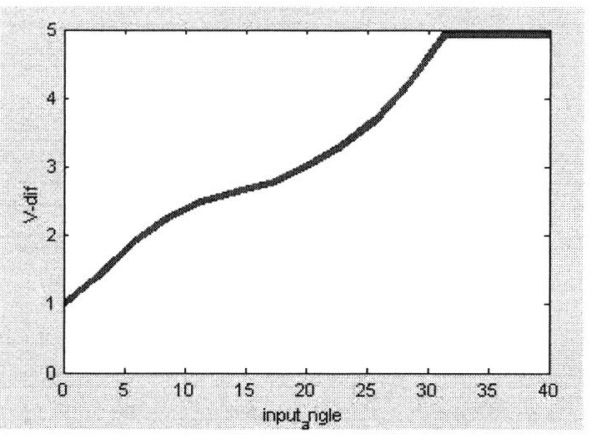

Fig. 10. Input /output relation of goal seeking FLC.

\section{Wall Following Behavior}

The wall following behavior is defined as to keep the same distance with the nearest wall. Fig. 11 depicts the design of wall following behavior. We calculated the difference of left and right distance obtained from the corresponding sensors. The distance difference in the left and right direction in two samples is taken as the input to the fuzzy logic controller. The output of fuzzy logic controller is the differential speed between two drive wheels. The membership function of wall following behavior is shown in Fig.12. Following this definition, we designed three fuzzy rules to achieve the wall following behavior.

a. If the differential distance is big, then the differential speed is big.

b. If the differential distance is medium, then the differential speed is medium.

c. If the differential distance is small, then the differential speed is zero.

When the differential distance is bigger, the differential speed should be larger. The input and output relation of the wall following FLC is shown in Fig. 13. 


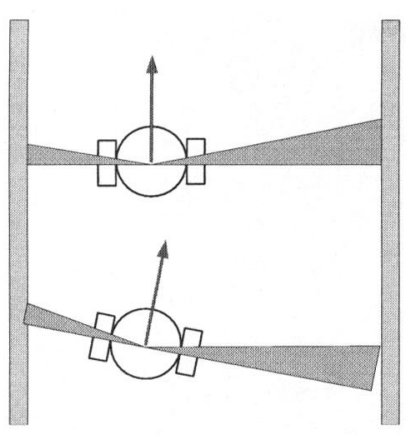

Fig.11. Design of wall-following behavior.

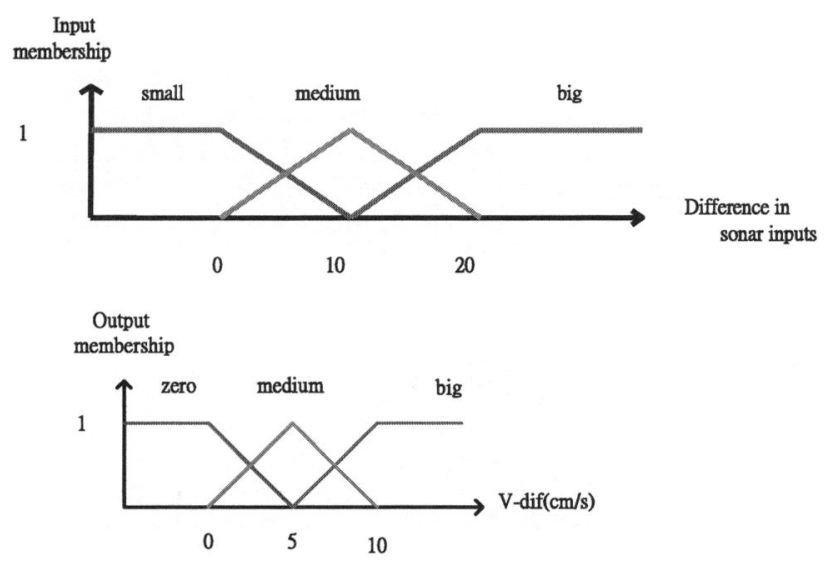

Fig.12. Membership function of wall following FLC.

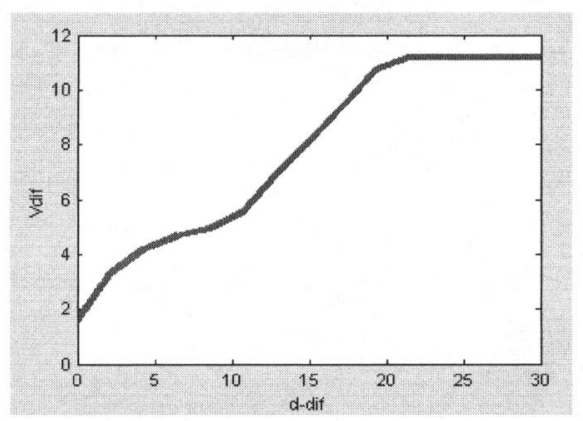

Fig.13. Input /output relation of wall following FLC.

\section{FUZZY-NEURO BEHABIOR FUSION}

The concept of pattern recognition was adopted for behavior fusion design. It is utilized to map the environmental pattern, obtained from ultrasonic sensors onboard the robot, to the fusion weight of three navigation behaviors. Considering that "when the environment information of surroundings is similar, the fusion weight of navigation behaviors should be similar too," we use the Fuzzy Kohonen Clustering Network (FKCN) [13-14] to recognize the environment. Fig.14 illustrates the structure of the fuzzy-neuro network for behavior fusion. In the first layer of he network, the quantized value of distance is regarded as the inputs of FKCN. In the second layer, we calculate the degree of difference between the current and the prototype pattern. If the input current environment pattern is not similar with the prototype pattern, the degree of difference becomes larger. In the third layer of FKCN, we obtain the membership or degree of similarity to the prototype pattern, from which the current fusion weights are determined. The Prototype patterns define the basic situation of environment as shown in Fig.15. In this design, eight prototype patterns are selected to represent typical situations for indoor navigation. Table. 1 shows the behavior fusion weight corresponding to each prototype pattern. In the design phase, one needs to assign the default fusion weight in the FKCN for distance calculation. In application, as the robot obtains current sensor data, the FKCN can find out the fusion weight corresponding to the immediate environment. FKCN has the capacity to generalize these prototype patterns to all situations that may happen to the robot duration navigation by giving the proper fusion weights. Fig. 16 illustrates the structure of behavior fusion.

Note that the ultrasonic distance data are quantized to five levels before sent to the FKCN.

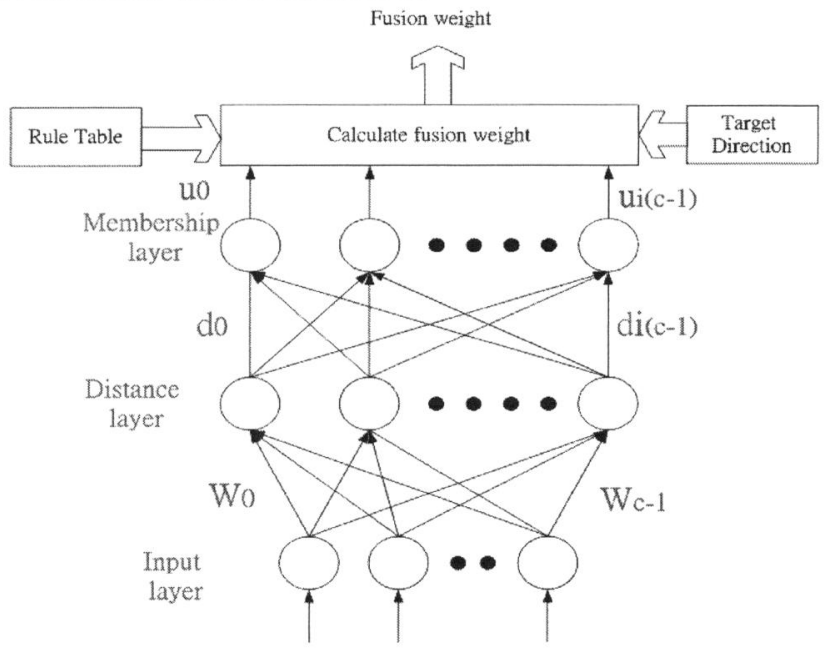

Fig.14. The FKCN for fusion weights determination.
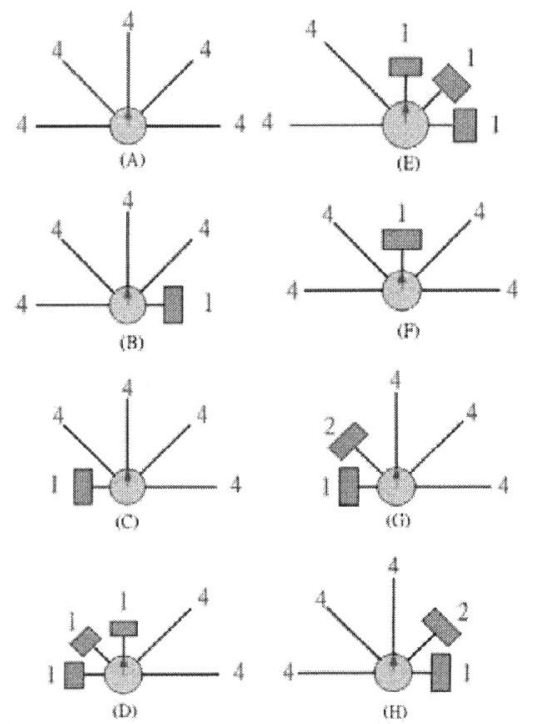

Fig.15. The prototype patterns. 
Table.1 Prototype patterns and fusion weights

\begin{tabular}{|c|c|c|c|c|c|c|c|c|c|c|c|c|c|c|c|c|c|c|c|}
\hline \multirow{3}{*}{\multicolumn{5}{|c|}{$\begin{array}{c}\text { IF-Part } \\
\text { prototype pattern }\end{array}$}} & \multicolumn{15}{|c|}{ THEN-Part(behavior fusion weight) } \\
\hline & & & & & \multicolumn{3}{|c|}{$\operatorname{din}=1$} & \multicolumn{3}{|c|}{$d i x=2$} & \multicolumn{3}{|c|}{ dir=3 } & \multicolumn{3}{|c|}{ dir $=4$} & \multicolumn{3}{|c|}{ dir $=5$} \\
\hline & & & & & & \multirow{2}{*}{ Wo $_{0}$} & & \multirow{2}{*}{$\begin{array}{c}\mathbf{W}_{\mathbf{g}} \\
1\end{array}$} & & & & & & \multirow{2}{*}{$\begin{array}{c}\mathrm{W}_{\mathrm{g}} \\
\mathrm{l}\end{array}$} & & & \multirow{2}{*}{\multicolumn{2}{|c|}{$\begin{array}{cc}W_{3} & W \\
1 & 0\end{array}$}} & \multirow{2}{*}{ - $\frac{W_{w}}{0}$} \\
\hline 4 & 4 & 4 & 4 & 4 & & & & & & & & & & & & & & & \\
\hline 4 & 4 & 4 & 4 & 1 & 1 & 0 & 0 & 1 & 0 & 0 & 1 & 0 & 0 & 0.7 & 0.3 & 0 & 0.5 & 0.5 & 0 \\
\hline & 4 & 4 & 4 & 4 & 0.5 & 0.5 & 0 & 0.7 & 0.3 & 0 & 1 & $\mathbf{0}$ & 0 & 1 & 0 & 0 & 1 & 0 & 0 \\
\hline 4 & 4 & 1 & 1 & 1 & 0.2 & 0.8 & 0 & 0.4 & 0.6 & 0 & 0 & 0.7 & 0.3 & 0 & 0.5 & 0.5 & 0.5 & 0.5 & 0 \\
\hline & 1 & 1 & 4 & 4 & 0.5 & 0.5 & 0 & 0.5 & 0.5 & 0 & 0 & 0.7 & 0.3 & 0.4 & 0.6 & 0 & 0.2 & 0.8 & 0 \\
\hline & 4 & 1 & 4 & 4 & 0.4 & 0.6 & 0 & 0.2 & 0.8 & 0 & 0 & 1 & 0 & 0.2 & 0.8 & 0 & 0.4 & 0.6 & 0 \\
\hline 1 & 2 & 4 & 4 & 4 & 0.4 & 0.6 & 0 & 0.5 & 0.5 & 0 & 0 & 0 & 1 & 0.3 & 0 & 0.7 & 0.7 & 0 & 0.3 \\
\hline & 4 & 4 & & & .7 & & J.3 & 0.3 & & 0.7 & 0 & & & 0.5 & & & & & \\
\hline
\end{tabular}

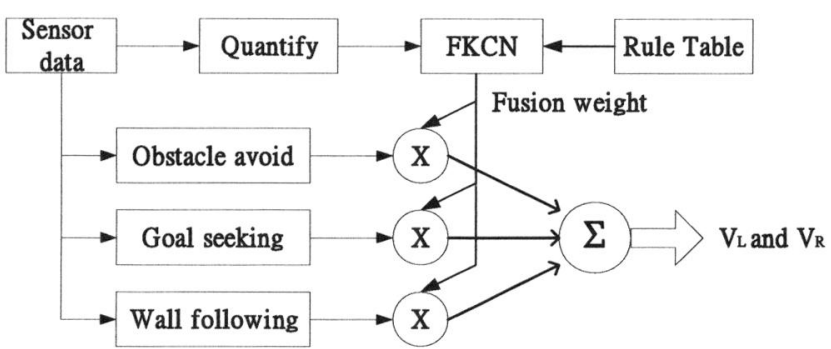

Fig.16. Block diagram of behavior fusion.

For ultrasonic sensor group $0,1,3,4$, formula (1) is used for quantization; where $d_{i}$ is the measured distance in $\mathrm{cm}$ of the $i$ th sensor group (direction). For the sensor group (2), formula(2) is used. The relative angle to the goal position also needs to be considered in navigation. The quantization of relative angle to the goal position is shown in Fig.17. We divide the angle to the goal into five sections. In Formula (3), five directions are assigned. The parameter dir represents the direction section.

$$
\begin{aligned}
& x_{i}=\left\{\begin{array}{lcc}
1 & \text { for } 0 \leq d_{i} \leq 90 \\
1+\left(d_{i}-90\right) / 60 & \text { for } & 90<d_{i} \leq 150 \\
2+\left(d_{i}-150\right) / 60 & \text { for } & 150<d_{i} \leq 210 \\
3+\left(d_{i}-210\right) / 60 & \text { for } & 210<d_{i} \leq 270 \\
4 & \text { for } & d_{i}>270
\end{array}\right. \\
& x_{i}=\left\{\begin{array}{lcc}
1 & \text { for } 0 \leq d_{i} \leq 120 \\
1+\left(d_{i}-120\right) / 60 & \text { for } 120<d_{i} \leq 180 \\
2+\left(d_{i}-180\right) / 60 & \text { for } & 180<d_{i} \leq 240 \\
3+\left(d_{i}-240\right) / 60 & \text { for } & 240<d_{i} \leq 300 \\
4 & \text { for } & d_{i}>300
\end{array}\right.
\end{aligned}
$$

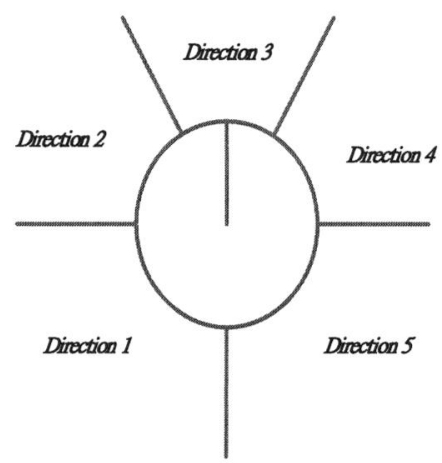

Fig. 17. Assignment of goal directions.

$$
\operatorname{dir}=\left\{\begin{array}{lll}
1 & \text { for } & 90 \leq \phi \leq 180 \\
2 & \text { for } & 30 \leq \phi<90 \\
3 & \text { for } & -30 \leq \phi<30 \\
4 & \text { for } & 30 \leq \phi<-90 \\
5 & \text { for } & -90 \leq \phi<-180
\end{array}\right.
$$

\section{EXPERIMENTAL RESULTS}

In this section, we present the experimental results of the proposed behavior fusion method. Fig. 18 illustrates the control structure of the mobile robot. Fig. 19 shows the experimental result of autonomous navigation in the lab environment. The robot navigated in a crowded room and passed a narrow door before it entered a corridor. There are several obstacles in the corridor as depicted in the figure. This experiment validates that the proposed behavior fusion is sufficient for the navigation in an indoor environment. In Fig.20, the recorded behavior fusion weights of three behaviors are illustrated. One can see that the weight of obstacle avoidance increased at sample instants 150, 190, and 230. It reflects that the mobile robot detected obstacles indicated as square shape in Fig. 19. At sample instants 90 and 170 , the fusion weights of goal seeking increased. It means that at these sample instants, the robot did not detect any obstacle in its heading direction, and it tried to regulate its traveling direction towards the goal. The second experiment tested the robot when it meets a dead end. The navigation result is shown in the Fig.21. One can see that the robot escaped from the dead end and traveled in the corridor as expected.

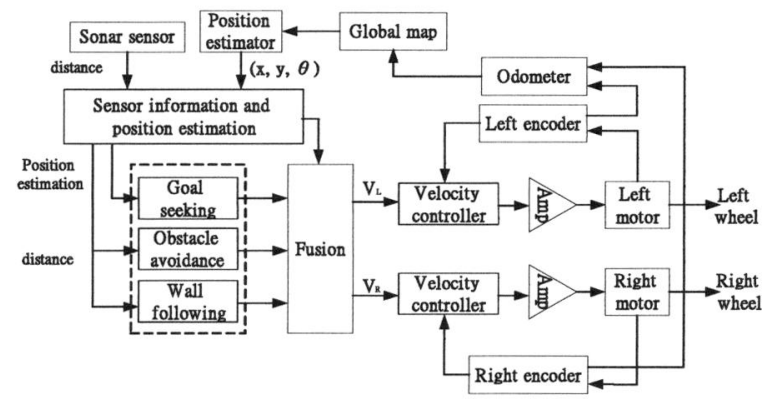

Fig.18. The structure of navigation system.

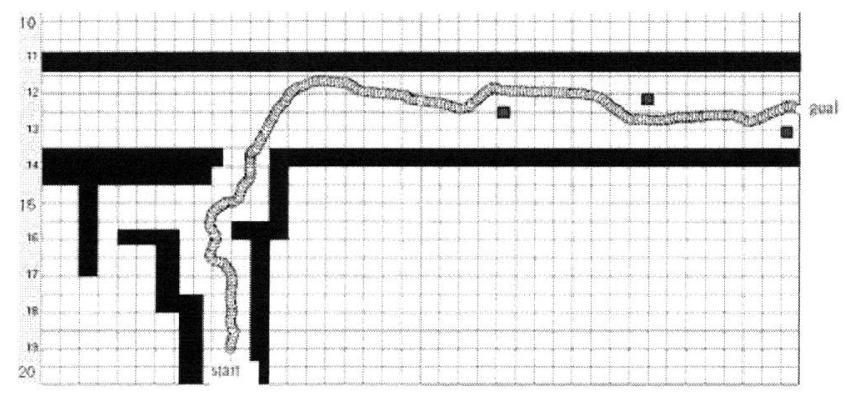

Fig.19. Experiment results of navigation through a narrow door and in the corridor. 


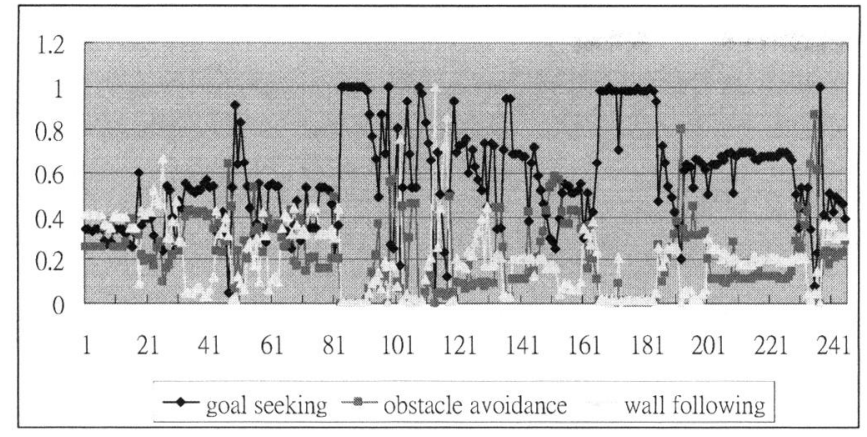

Fig.20. Recorded fusion weights of experiment of Fig. 19.

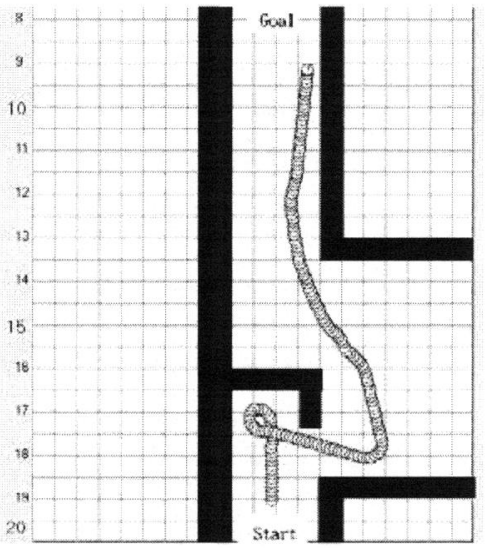

Fig.21. Experiment results of escaping from a dead-end.

\section{CONCLUSION}

This paper presents a behavior-fusion architecture for a mobile robot. This architecture successfully coordinates the output of three navigation behaviors by using the fuzzy-neuro pattern recognition network. Thanks to the autonomous behavior fusion design, the individual behavior can be implemented using simple fuzzy logic rules. The experimental results reveal the system can determine the proper fusion weight and response to the dynamic changing environment efficiently. However, it has been observed in the experiments that there are some errors at the end of path. This is mainly caused by the position estimation error of the mobile robot. In the future, we will add more accurate localization design to the robot and continue to investigate the behavior fusion method for more complex tasks.

\section{REFERENCES}

[1] R. A. Brooks. "A robust layered control system for a mobile robot," IEEE Journal of Robotics and Automation, vol.2, no.7, pp.14-23, 1986.

[2] M. J. Mataric, "Behavior-based control: examples from navigation, learning and group behavior," Journal of Experimental Theoretical Artificial Intelligence, Special Issue: Software Architecture Phys. Agents, vol. 9, pp.4654., 1997.
[3] S. G. Goodridge and R. C. Luo, "Fuzzy behavior fusion for reactive control of an autonomous mobile robot: MARGE," Proc. of 1994 IEEE International Conference on Robotics and Automation, 1994, pp. $1622-1627$.

[4] Wei Li and Xun Feng, "Behavior fusion for robot navigation in uncertain environments using fuzzy logic," Proc. of 1994 IEEE International Conference on Systems, Man, and Cybernetics, 1994, pp.1790-1796.

[5] C. Ye and N.H.C. Yung, "Vehicle navigation strategy based on behavior fusion," Proc. of 1997 IEEE International Conference on Systems, Man, and Cybernetics, 1997, pp. $3698-3703$.

[6] N.H.C. Yung and C. Ye, "Avoidance of moving obstacles through behavior fusion and motion prediction," 1998 IEEE International Conference on Systems, Man, and Cybernetics, pp. 3424 -3429, 1998.

[7] Cang Ye and Danwei Wang, "A novel behavior fusion method for the navigation of mobile robots," Proc. of 2000 IEEE International Conference on Systems, Man, and Cybernetics, 2000, pp.3526-3531.

[8] P. Rusu, E. M. Petriu, T. E. Whalen, and A. Cornell, and H. J.W. Spoelder, "Behavior-based neuro-fuzzy controller for mobile robot navigation," IEEE Transactions on Instrumentation and Measurement, Vol. 52, Issue 4, pp.1335 - 1340, 2003.

[9] Simon X. Yang, Hao Li and Max Meng. "Fuzzy control of a behavior-based mobile robot," The 12th IEEE International Conference on Fuzzy Systems, 2003, pp.319-324.

[10] X. Yang, M. Moallem, and R.V. Patel, “An improved fuzzy logic based navigation system for mobile robots," Proceedings of the 2003 IEEE/RSJ Intl. Conference on Intelligent Robots and Systems, Las Vegas, USA, 2003, pp.1709-1714.

[11] Simon X Yang, Hao Li, and Peter X. Liu, "An embedded fuzzy controller for a behavior-based mobile robot with guaranteed performance," IEEE Transactions on fuzzy systems, vol. 12, no.4, pp.436-446, 2004

[12] S. Khatoon, "Behavior coordination of autonomous mobile robot navigation by neuro-fuzzy system," Proceedings of the IEEE 31st Annual Northeast Bioengineering Conference, 2005, pp.58 - 62.

[13] T . Huntsberger and P. Ajjimarangsee, "Parallel Selforganizing Feature Maps for Unsupervised Pattern Recognition," Int'l. J. General Systems, vol. 16, no.4, pp.357-372, 1990.

[14] K.T. Song and L.H. Sheen, "Heuristic Fuzzy-neuro Network and its Application to Reactive Navigation of a Mobile Robot,' Fuzzy Sets and Systems, Vol.110, No.3, pp. 331-340, 2000. 